\title{
SIMPLE NECESSARY CONDITIONS FOR HADAMARD FACTORIZABILITY OF HURWITZ POLYNOMIALS*
}

\author{
STANISŁAW BIAŁAS ${ }^{\dagger}$ AND MICHAŁ GÓRA
}

\begin{abstract}
In this paper, we focus the attention on the Hadamard factorization problem for Hurwitz polynomials. We give a new necessary condition for Hadamard factorizability of Hurwitz stable polynomials of degree $n \geq 4$ and show that for $n=4$ this condition is also sufficient. The effectiveness of the result is illustrated during construction of examples of stable polynomials that are not Hadamard factorizable.
\end{abstract}

Key words. Hadamard factorization, Hadamard product of polynomials, Hurwitz stable polynomials.

AMS subject classifications. 26C10, 30C15, 93D99.

1. Introduction. A problem of the existence of a Hadamard factorization for a given Hurwitz stable polynomial has been taken by many authors. Recall that a Hurwitz stable polynomial of degree $n \geq 1$ has a Hadamard factorization if it is a Hadamard product of two Hurwitz stable polynomials of degree $n$. It is known that every stable polynomial of degree $n \leq 3$ admits a Hadamard factorization (see Garloff and Shrinivasan [5]) and that for every $n \geq 4$ there exists an $n$-th degree stable polynomial which is not Hadamard factorizable (see Białas and Góra [2]). Some conditions for the existence of a Hadamard factorization can be found in Loredo-Villalobos and Aguirre-Hernández [8, 9], but these conditions cannot be effectively applied in practice. In turn, some topological properties of the entire family of polynomials admitting a Hadamard factorization can be found in Aguirre-Hernández et al. [1].

Note also, that there are some issues in which polynomials having a Hadamard factorization play an important role. In [3], the authors have considered the stability problem for the generalized Hadamard product of polynomials (recall that the generalized Hadamard product $f \bullet g$ of polynomials $f$ of degree $m$ and $g$ of degree $n \geq m$ is a set consisting of polynomials of degree $m$ which are defined as the Hadamard products of the polynomial $f$ and some other $n-m+1$ polynomials formed from the polynomial $g$ ). It was shown, among others, that if $f$ and $g$ are Hurwitz stable, then $f \bullet g$ is quasi-stable (i.e. all zeros of every polynomial belonging to $f \bullet g$ have non-positive real parts). If, additionally, $f$ has a Hadamard factorization, then $f \bullet g$ occurs to be Hurwitz stable. This shows that the Hadamard factorization problem has both theoretical and applied significance.

In this work, we develop the idea used in our recent paper [2]. We give a new necessary condition for Hadamard factorizability of a polynomial and show that for $n=4$ this condition is also sufficient. The effectiveness of the result is illustrated during construction of examples of stable polynomials that are not Hadamard factorizable.

* Received by the editors on March 3, 2021. Accepted for publication on October 9, 2021. Handling Editor: Froilán Dopico. Corresponding Author: Michał Góra

$\dagger$ The School of Banking and Management, ul. Armii Krajowej 4, 30-150 Kraków, Poland (sbialas@agh.edu.pl)

¥AGH University of Science and Technology, Faculty of Applied Mathematics, al. Mickiewicza 30, 30-059 Kraków, Poland (gora@agh.edu.pl). 
2. Preliminary results. Let us now introduce the notations and remind some results which will be used in the sequel.

2.1. Basic notations. We use standard notation: $\mathbb{R}$ and $\mathbb{R}^{n \times n}$ stand for the set of real numbers and for the set of real matrices of order $n \times n$, respectively; $\pi_{n}^{+}$denotes the family of $n$-th degree polynomials with positive coefficients.

2.2. Stable polynomials. A polynomial $f \in \pi_{n}^{+}(n \geq 1)$,

$$
f(s)=a_{0}+a_{1} s+\ldots+a_{n-1} s^{n-1}+a_{n} s^{n},
$$

is Hurwitz stable (or shortly stable) if all its zeros have negative real parts. It is well known (and easily verified) that a necessary condition for the stability of a real polynomial is that its coefficients are all of the same sign; without losing generality we will assume in the sequel that they are positive. The entire family of Hurwitz stable polynomials of degree $n$ with positive coefficients will be denoted by $\mathcal{H}_{n}^{+}$.

Let $\triangle_{i}(f)$ denote the $i$-th leading principal minor of the Hurwitz matrix $H_{f} \in \mathbb{R}^{n \times n}$ associated with polynomial (1):

$$
H_{f}=\left[\begin{array}{cccccc}
a_{n-1} & a_{n} & 0 & 0 & \ldots & 0 \\
a_{n-3} & a_{n-2} & a_{n-1} & a_{n} & \ldots & 0 \\
a_{n-5} & a_{n-4} & a_{n-3} & a_{n-2} & \ldots & 0 \\
\vdots & \vdots & a_{n-5} & a_{n-4} & \ldots & 0 \\
\vdots & \vdots & \vdots & \vdots & \ddots & \vdots \\
0 & 0 & 0 & 0 & \ldots & a_{0}
\end{array}\right]
$$

in particular $\triangle_{1}(f)=a_{n-1}$ and $\triangle_{n}(f)=\operatorname{det} H_{f}=a_{0} \triangle_{n-1}(f)$. It follows from the Routh-Hurwitz stability criterion (see, e.g., Gantmacher [4]) that polynomial (1) with positive coefficients is stable if and only if $\triangle_{i}(f)>0$, for $i=1,2, \ldots, n-1$. Among many other properties of the Hurwitz matrix, one can also find the following given by Kemperman [7] (see Theorem 2 therein).

TheOREM 1. If $f \in \mathcal{H}_{n}^{+}$, then every square submatrix of the Hurwitz matrix $H_{f}$ has positive determinant if and only if all its diagonal elements are positive.

Suppose now that $n \geq 4$ and that $\left\{H_{1}, \ldots, H_{m_{n}}\right\} \subset \mathbb{R}^{3 \times 3}$ is a set of all $3 \times 3$ submatrices of the form:

$$
H_{f, k}=\left[\begin{array}{ccc}
h_{11}^{(k)} & h_{12}^{(k)} & 0 \\
h_{21}^{(k)} & h_{22}^{(k)} & h_{23}^{(k)} \\
0 & h_{32}^{(k)} & h_{33}^{(k)}
\end{array}\right] \quad \text { with } h_{i j}^{(k)}>0
$$

of the Hurwitz matrix $H_{f}$. Entries $h_{i j}^{(k)}$ occurring in (3) depend on the polynomial $f$ but, to simplify the notation, throughout this paper we do not make this dependence explicit.

ExAmPle 1. For $n=4$ we have $m_{4}=2$ and

$$
H_{f, 1}=\left[\begin{array}{ccc}
a_{3} & a_{4} & 0 \\
a_{1} & a_{2} & a_{3} \\
0 & a_{0} & a_{1}
\end{array}\right] \text { and } H_{f, 2}=\left[\begin{array}{ccc}
a_{3} & a_{4} & 0 \\
a_{1} & a_{2} & a_{4} \\
0 & a_{0} & a_{2}
\end{array}\right] \text {. }
$$


For $n=5$ we have $m_{5}=4$ and

$$
\begin{array}{lll}
H_{f, 1}= & {\left[\begin{array}{ccc}
a_{3} & a_{4} & 0 \\
a_{1} & a_{2} & a_{4} \\
0 & a_{0} & a_{2}
\end{array}\right],} & H_{f, 2}=\left[\begin{array}{ccc}
a_{3} & a_{5} & 0 \\
a_{1} & a_{3} & a_{4} \\
0 & a_{1} & a_{2}
\end{array}\right], \\
H_{f, 3}=\left[\begin{array}{ccc}
a_{2} & a_{4} & 0 \\
a_{0} & a_{2} & a_{4} \\
0 & a_{0} & a_{2}
\end{array}\right], & H_{f, 4}=\left[\begin{array}{ccc}
a_{2} & a_{5} & 0 \\
a_{0} & a_{3} & a_{4} \\
0 & a_{1} & a_{2}
\end{array}\right] .
\end{array}
$$

Theorem 1 allows us to conclude that if polynomial (1) of degree $n \geq 4$ is stable then

$$
h_{11}^{(k)} h_{22}^{(k)}-h_{12}^{(k)} h_{21}^{(k)}>0, \quad h_{22}^{(k)} h_{33}^{(k)}-h_{23}^{(k)} h_{32}^{(k)}>0,
$$

and

$$
h_{11}^{(k)} h_{22}^{(k)} h_{33}^{(k)}-h_{11}^{(k)} h_{23}^{(k)} h_{32}^{(k)}-h_{12}^{(k)} h_{21}^{(k)} h_{33}^{(k)}>0,
$$

for $k=1, \ldots, m_{n}$. These inequalities will play a key role in our further considerations.

2.3. Polynomials admitting a Hadamard factorization. Together with polynomial (1), we will consider a polynomial $g \in \pi_{n}^{+}$:

$$
g(s)=b_{0}+b_{1} s+\ldots+b_{n-1} s^{n-1}+b_{n} s^{n},
$$

their Hadamard product $f \circ g \in \pi_{n}^{+}$defined as an element-wise multiplication, that is,

$$
(f \circ g)(s)=a_{0} b_{0}+a_{1} b_{1} s+\ldots+a_{n-1} b_{n-1} s^{n-1}+a_{n} b_{n} s^{n},
$$

and their Hadamard quotient $f \diamond g \in \pi_{n}^{+}$defined as an element-wise division, that is,

$$
(f \diamond g)(s)=\frac{a_{0}}{b_{0}}+\frac{a_{1}}{b_{1}} s+\ldots+\frac{a_{n-1}}{b_{n-1}} s^{n-1}+\frac{a_{n}}{b_{n}} s^{n} .
$$

Garloff and Wagner [6] proved that the Hadamard product of two real Hurwitz stable polynomials is again Hurwitz stable, and thus it seems to be quite natural to say that the polynomial $f \in \mathcal{H}_{n}^{+}$has a Hadamard factorization (or is Hadamard factorizable) if there exist two polynomials $f_{1}, f_{2} \in \mathcal{H}_{n}^{+}$such that $f=f_{1} \circ f_{2}$. Equivalently, the polynomial $f \in \mathcal{H}_{n}^{+}$has a Hadamard factorization if there exists a polynomial $g \in \mathcal{H}_{n}^{+}$ such that $f \diamond g \in \mathcal{H}_{n}^{+}$. A stable polynomial which is not Hadamard factorizable is said to be Hadamard irreducible.

3. Main results. Let $f \in \pi_{n}^{+}$and let $w_{f, 1}^{(k)}, w_{f, 2}^{(k)}$ be positive numbers given by:

$$
w_{f, 1}^{(k)}=\frac{h_{21}^{(k)} h_{12}^{(k)}}{h_{11}^{(k)} h_{22}^{(k)}}, \quad w_{f, 2}^{(k)}=\frac{h_{32}^{(k)} h_{23}^{(k)}}{h_{22}^{(k)} h_{33}^{(k)}},
$$

for $k=1, \ldots, m_{n}$. We start with the following simple observation.

Lemma 2. Let $n \geq 4$.

(a) If $f \in \mathcal{H}_{n}^{+}$, then for $k=1, \ldots, m_{n}$

$$
w_{f, 1}^{(k)}<1, \quad w_{f, 2}^{(k)}<1, \quad w_{f, 1}^{(k)}+w_{f, 2}^{(k)}<1 .
$$


(b) If $f \in \mathcal{H}_{n}^{+}$and $g \in \pi_{n}^{+}$are such that $f \diamond g \in \mathcal{H}_{n}^{+}$, then for $k=1, \ldots, m_{n}$

$$
w_{f, 1}^{(k)}<w_{g, 1}^{(k)}, \quad w_{f, 2}^{(k)}<w_{g, 2}^{(k)},
$$

and

$$
w_{f, 1}^{(k)} / w_{g, 1}^{(k)}+w_{f, 2}^{(k)} / w_{g, 2}^{(k)}<1 .
$$

Proof. The above inequalities follow from the stability of $f$ and $f \diamond g$ : conditions (8) are equivalent to (4)-(5) and conditions (9)-(10) follow from (8) and from the identities:

$$
w_{f \diamond g, 1}^{(k)}=w_{f, 1}^{(k)} / w_{g, 1}^{(k)} \quad \text { and } \quad w_{f \diamond g, 2}^{(k)}=w_{f, 2}^{(k)} / w_{g, 2}^{(k)} .
$$

3.1. A necessary condition for Hadamard factorizability of real polynomials. We are now ready to prove the main result of this section.

THEOREM 3. Let $n \geq 4$. If a polynomial $f \in \pi_{n}^{+}$has a Hadamard factorization, then

$$
\Delta_{k}(f)=\left(w_{f, 1}^{(k)}-w_{f, 2}^{(k)}\right)^{2}-2\left(w_{f, 1}^{(k)}+w_{f, 2}^{(k)}\right)+1>0,
$$

for $k=1, \ldots, m_{n}$

Proof. It follows from the assumption that there exists a polynomial $g \in \mathcal{H}_{n}^{+}$for which the polynomial $f \diamond g$ is stable. Then, by Lemma 2 , we conclude that for any fixed $k \in\left\{1, \ldots, m_{n}\right\}$ it holds

$$
w_{g, 1}^{(k)}+w_{g, 2}^{(k)}<1
$$

and

$$
w_{f, 1}^{(k)} / w_{g, 1}^{(k)}+w_{f, 2}^{(k)} / w_{g, 2}^{(k)}<1 .
$$

In other words, the Hadamard factorizability of $f$ implies that there exist $x_{1}>1$ and $x_{2}>1$ satisfying the following system of inequalities:

$$
\left\{\begin{array}{c}
\frac{1}{x_{1}}+\frac{1}{x_{2}}<1 \\
b_{1} x_{1}+b_{2} x_{2}<1
\end{array},\right.
$$

where, to simplify the notations, we put $b_{i}=w_{f, i}^{(k)}$, for $i=1,2$. By Lemma 2 , we know that $b_{1}, b_{2} \in(0,1)$. It is easy to note (see Fig. 1) that system of inequalities (12) has a solution if and only if the system:

$$
\left\{\begin{array}{r}
\frac{1}{x_{1}}+\frac{1}{x_{2}}=1 \\
b_{1} x_{1}+b_{2} x_{2}=1 \\
x_{1}>1, x_{2}>1
\end{array},\right.
$$

has two different solutions $\left(x_{1}^{\prime}, x_{2}^{\prime}\right)$ and $\left(x_{1}^{\prime \prime}, x_{2}^{\prime \prime}\right)$. This condition holds, in turn, if and only if the equation:

$$
b_{2} x_{2}^{2}+\left(b_{1}-b_{2}-1\right) x_{2}+1=0,
$$

has two different solutions $x_{2}^{\prime}>1$ and $x_{2}^{\prime \prime}>1$. This, by (8), is equivalent to (11) completing the proof.

From Theorem 3 one can draw the following sufficient condition for Hadamard irreducibility of a polynomial.

Conclusion 1. Let $n \geq 4$. If $f \in \mathcal{H}_{n}^{+}$and for some $k \in\left\{1, \ldots, m_{n}\right\}$ :

$$
\Delta_{k}(f) \leq 0,
$$

where $\Delta_{k}(f)$ is as in (11), then $f$ is Hadamard irreducible. 


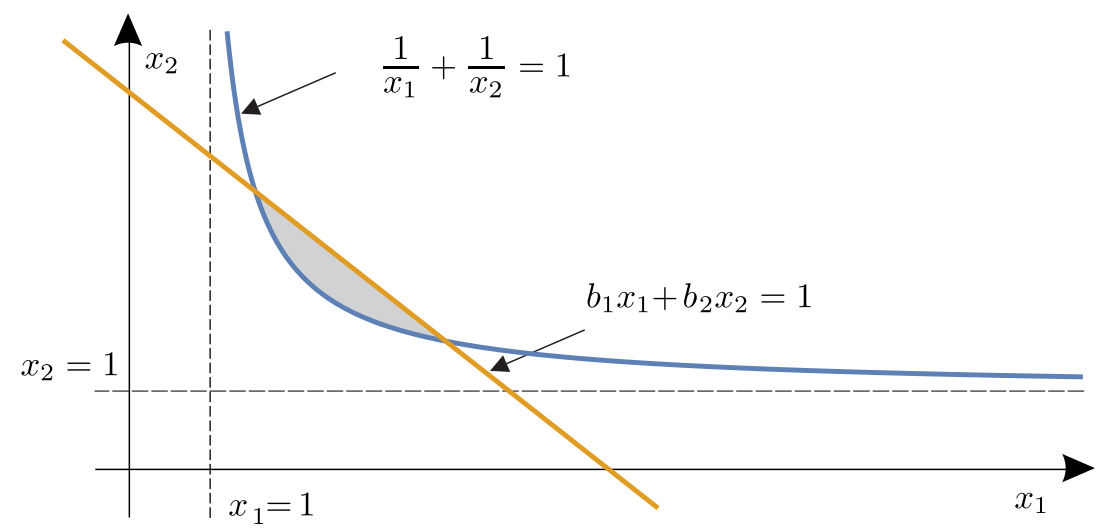

FIGURE 1. Solutions to system of inequalities (12) filled with gray color.

3.2. How to get a Hadamard irreducible polynomial? Garloff and Srinivasan considered in [5] the Hadamard factorization problem and gave, among others, an example of the Hadamard irreducible polynomial of degree $n=4$. Now, using Theorem 3 and Conclusion 1, we will show a fairly universal way of construction of further examples of Hadamard irreducible polynomials of arbitrary degree $n \geq 4$. First, we need to introduce some additional notations.

Let, for $f \in \mathcal{H}_{n}^{+}$and $a>0, F_{a} \in \pi_{n+1}^{+}$be a polynomial of the form:

$$
F_{a}(s)=a s^{n+1}+f(s),
$$

and let $A, A_{1}, \ldots, A_{m_{n+1}} \subset \mathbb{R}$ be sets defined as follows:

$$
A=\left\{a>0: F_{a} \in \mathcal{H}_{n+1}^{+}\right\},
$$

and, for $k=1, \ldots, m_{n+1}$,

$$
A_{k}=\left\{a>0: \Delta_{k}\left(F_{a}\right) \leq 0\right\}
$$

The following theorem holds.

TheOREM 4. Let $n \geq 4$ and let $f \in \mathcal{H}_{n}^{+}$. Under the above notations, if for some $k \in\left\{1, \ldots, m_{n+1}\right\}$ we have

$$
A \cap A_{k} \neq \emptyset,
$$

then for every $a \in A \cap A_{k}$ the polynomial $F_{a}$ is stable and Hadamard irreducible.

Proof. The thesis is a simple consequence of Conclusion 1.

It is known (see, e.g., Lemma 3.1 in Białas and Góra [3]) that if $f \in \mathcal{H}_{n}^{+}$then there exists $a^{*}>0$ such that $F_{a} \in \mathcal{H}_{n+1}^{+}$for every $a \in\left(0, a^{*}\right)$. This means that the set $A$ is always non-empty. On the other hand, one can show that if the polynomial $f$ is stable, then sets $A_{1}, \ldots, A_{m_{n+1}}$ are all separated from zero and, hence, it is possible that in some cases assumption (15) of Theorem 4 will not be satisfied.

3.2.1. A numerical example. Consider a Hurwitz stable polynomial $f \in \pi_{3}^{+}$of the form:

$$
f(s)=s^{3}+3 s^{2}+s+1,
$$


and let, for $a>0$,

$$
F_{a}(s)=a s^{4}+s^{3}+3 s^{2}+s+1 .
$$

It follows from the Routh-Hurwitz stability criterion that the polynomial $F_{a}$ is stable if and only if

$$
0 \leq a<2
$$

On the other hand, submatrices (3) of the Hurwitz matrix $H_{F_{a}}$ have the form (see Example 1):

$$
H_{F_{a}, 1}=\left[\begin{array}{ccc}
1 & a & 0 \\
1 & 3 & 1 \\
0 & 1 & 1
\end{array}\right] \text { and } H_{F_{a}, 2}=\left[\begin{array}{ccc}
1 & a & 0 \\
1 & 3 & a \\
0 & 1 & 3
\end{array}\right]
$$

Thus, according to (7),

$$
w_{F_{a}, 1}^{(1)}=\frac{a}{3}, w_{F_{a}, 2}^{(1)}=\frac{1}{3} \quad \text { and } \quad w_{F_{a}, 1}^{(2)}=\frac{a}{3}, w_{F_{a}, 2}^{(2)}=\frac{a}{9} .
$$

Simple calculations show that the condition $\Delta_{1}\left(F_{a}\right) \leq 0$ is satisfied if and only if

$$
a_{\min } \leq a \leq a_{\max }
$$

where $a_{\min }=4-2 \sqrt{3} \approx 0.5359$ and $a_{\max }=4+2 \sqrt{3} \approx 7.4641$. It follows from Theorem 4 that for every $a \in\left[a_{\min }, 2\right)$ the polynomial $F_{a}$ is Hadamard irreducible; for $a=1$ we get a Hadamard irreducible polynomial $g(s)=s^{4}+s^{3}+3 s^{2}+s+1$ obtained previously by Garloff and Srinivasan [5].

To construct a Hadamard irreducible polynomial of degree $n=5$, we put $a=3 / 2$ (unfortunatly, for $a=1$ assumption (15) of Theorem 4 is not satisfied) and repeat the above reasoning. As previously, we begin with considering the polynomial:

$$
G_{a}(s)=a s^{5}+3 / 2 s^{4}+s^{3}+3 s^{2}+s+1,
$$

and showing that it is stable if and only if

$$
0 \leq a<a^{*}=-3 / 2+\sqrt{3} \approx 0.2320 .
$$

One of the submatrices (3) of the Hurwitz matrix $H_{G_{a}}$ has the form:

$$
H_{G_{a}, 1}=\left[\begin{array}{ccc}
1 & a & 0 \\
1 & 1 & 3 / 2 \\
0 & 1 & 3
\end{array}\right]
$$

and the condition $\Delta_{1}\left(G_{a}\right) \leq 0$ is satisfied if and only if

$$
a_{\min } \leq a \leq a_{\max },
$$

where $a_{\min }=3 / 2-\sqrt{2} \approx 0.0858$ and $a_{\max }=3 / 2+\sqrt{2} \approx 2.9142$. Combining (18) and (19), we conclude that for every $a \in\left[a_{\min }, a^{*}\right)$ the polynomial $G_{a}$ is Hadamard irreducible; taking for example $a=1 / 5$ we get a quintic polynomial:

$$
h(s)=1 / 5 s^{5}+3 / 2 s^{4}+s^{3}+3 s^{2}+s+1 .
$$


When considering the submatrix:

$$
\left[\begin{array}{ccc}
1 / 5 & a & 0 \\
1 & 3 / 2 & 1 / 5 \\
0 & 1 & 1
\end{array}\right],
$$

of the Hurwitz matrix for the polynomial $H_{a}(s)=a s^{6}+h(s)$ and performing similar calculations as above, we show that the polynomial:

$$
k(s)=1 / 5 s^{6}+1 / 5 s^{5}+3 / 2 s^{4}+s^{3}+3 s^{2}+s+1,
$$

is stable but not Hadamard factorizable.

3.3. A necessary and sufficient condition for Hadamard factorizability of fourth-degree real polynomials. In this last part of our work, we would like to focus the attention on polynomials of degree $n=4$. We will show that in that case a necessary condition for the Hadamard factorizability of a polynomial given in Theorem 3 is also sufficient.

To do this, let $f, g \in \pi_{4}^{+}$be two polynomials of the form:

$$
\begin{aligned}
& f(s)=a_{4} s^{4}+a_{3} s^{3}+a_{2} s^{2}+a_{1} s+a_{0}, \\
& g(s)=b_{4} s^{4}+b_{3} s^{3}+b_{2} s^{2}+b_{1} s+b_{0},
\end{aligned}
$$

and let

$$
w_{f, 1}=\frac{a_{1} a_{4}}{a_{2} a_{3}}, \quad w_{f, 2}=\frac{a_{0} a_{3}}{a_{2} a_{1}}, \quad w_{g, 1}=\frac{b_{1} b_{4}}{b_{2} b_{3}}, \quad w_{g, 2}=\frac{b_{0} b_{3}}{b_{2} b_{1}} .
$$

REMARK 1. It follows from the Routh-Hurwitz stability criterion that

(a) $f \in \pi_{4}^{+}$is stable if and only if $w_{f, 1}+w_{f, 2}<1$;

(b) for $f, g \in \pi_{4}^{+}, f \diamond g$ is stable if and only if $w_{f, 1} / w_{g, 1}+w_{f, 2} / w_{g, 2}<1$.

The following theorem holds.

Theorem 5. A polynomial $f \in \mathcal{H}_{4}^{+}$is Hadamard factorizable if and only if

$$
\left(w_{f, 1}-w_{f, 2}\right)^{2}-2\left(w_{f, 1}+w_{f, 2}\right)+1>0,
$$

where $w_{f, 1}$ and $w_{f, 2}$ are as in (20).

Before proceeding to the proof, we need an auxiliary lemma.

Lemma 6. Suppose that $0<b_{1}<1,0<b_{2}<1$ and that

$$
\left(b_{1}-b_{2}\right)^{2}-2\left(b_{1}+b_{2}\right)+1>0 .
$$

Then

1. there exist $x_{1}>1$ and $x_{2}>1$ satisfying the system of inequalities:

$$
\left\{\begin{array}{c}
b_{1} x_{1}+b_{2} x_{2}<1 \\
\frac{1}{x_{1}}+\frac{1}{x_{2}}<1
\end{array} ;\right.
$$


2. it holds

$$
\sqrt{b_{1}}+\sqrt{b_{2}}<1
$$

Proof. The first part of the thesis follows from the proof of Theorem 3 where the equivalence of conditions (12) and (14) was derived.

To prove the second part note that the first one implies that $b_{1}+b_{2}<1$. Then, (24) is a simple consequence of the following equality:

$$
\left(b_{1}-b_{2}\right)^{2}-2\left(b_{1}+b_{2}\right)+1=\left(1-\left(\sqrt{b_{1}}+\sqrt{b_{2}}\right)^{2}\right)\left(1-b_{1}-b_{2}+2 \sqrt{b_{1} b_{2}}\right) .
$$

Proof of Theorem 5. The necessity of (21) for the Hadamard factorizability of $f$ follows from Theorem 3. To prove the sufficiency, we will show that condition (21) implies that there exists a polynomial $g \in \mathcal{H}_{4}^{+}$ such that $f \diamond g \in \mathcal{H}_{4}^{+}$.

It follows from (21) and from Lemma 6 that there exist $s_{1}>1, s_{2}>1$ such that

$$
\left\{\begin{array}{c}
w_{f, 1} \cdot s_{1}+w_{f, 2} \cdot s_{2}<1 \\
\frac{1}{s_{1}}+\frac{1}{s_{2}}<1
\end{array} .\right.
$$

Let, for any solutions $s_{1}>1$ and $s_{2}>1$ of system $(25)$,

$$
g(s)=s^{4}+2 s^{3}+s^{2}+\frac{2}{s_{1}} s+\frac{1}{s_{1} s_{2}} .
$$

According to (20), we have $w_{g, 1}=1 / s_{1}$ and $w_{g, 2}=1 / s_{2}$. It follows from Remark 1 (a) and from (25) that polynomial (26) is stable. Besides, as follows from (25),

$$
w_{f, 1} / w_{g, 1}+w_{f, 2} / w_{g, 2}<1
$$

It means, by Remark $1(\mathrm{~b})$, that $f \diamond g$ is stable too. This completes the proof.

As a conclusion, we present the following theorem collecting the necessary and sufficient conditions for Hadamard factorizability of polynomials of degree $n=4$ derived in this section.

THEOREM 7. For $f \in \mathcal{H}_{4}^{+}$, the following conditions are equivalent:

(a) the polynomial $f(s)=a_{4} s^{4}+a_{3} s^{3}+a_{2} s^{2}+a_{1} s+a_{0}$ is Hadamard factorizable;

(b) it holds

$$
\left(w_{f, 1}-w_{f, 2}\right)^{2}-2\left(w_{f, 1}+w_{f, 2}\right)+1>0
$$

(c) it holds

$$
\sqrt{w_{f, 1}}+\sqrt{w_{f, 2}}<1
$$

(d) it holds

$$
\frac{a_{0} a_{3}}{a_{1}}<\left(\sqrt{\frac{a_{1} a_{4}}{a_{3}}}-\sqrt{a_{2}}\right)^{2} ;
$$

(e) the polynomial $g(s)=\sqrt{a_{4}} s^{4}+\sqrt{a_{3}} s^{3}+\sqrt{a_{2}} s^{2}+\sqrt{a_{1}} s+\sqrt{a_{0}}$ is stable. 
Proof. The equivalence $(a) \Leftrightarrow(b)$ follows from Theorem 5 , the implication $(b) \Rightarrow(c)$ follows from Lemma 6 , the equivalence $(c) \Leftrightarrow(d)$ is straightforward and follows from the stability of $f$, the equivalence $(c) \Leftrightarrow(e)$ follows from Remark 1 and from the identities $w_{g, 1}=\sqrt{w_{f, 1}}$ and $w_{g, 2}=\sqrt{w_{f, 2}}$, and the last implication $(e) \Rightarrow(a)$ follows from the obvious equality $f=g \circ g$. This completes the proof.

Let us point out that alternative proofs of two equivalences stated in Conclusion 7 can be found in some earlier works: the equivalence $(a) \Leftrightarrow(d)$ was previously proven by Loredo-Villalobos and Aguirre-Hernández [8] (see Lemma 3 therein) and $(a) \Leftrightarrow(e)$ by Aguirre-Hernández et al. [1] (see Theorem 7 therein).

\section{REFERENCES}

[1] B. Aguirre-Hernández, E.C. Díaz-González, C.A. Loredo-Villalobos, and F.R. García-Sosa. Properties of the set of Hadamardized Hurwitz polynomials. Math. Probl. Eng., Article ID 695279, 8 pages, 2015, doi: 10.1155/2015/695279.

[2] S. Białas and M. Góra. On the existence of Hurwitz polynomials with no Hadamard factorization. Electron. J. Linear Algebra, 36:210-213, 2020.

[3] S. Białas and M. Góra. The generalized Hadamard product of polynomials and its stability. Linear Multilinear Algebra, 69(7):1338-1351, 2021.

[4] F.R. Gantmacher. The Theory of Matrices, vol. II, Chelsea Publ., New York, 1959.

[5] J. Garloff and B. Shrinivasan. The Hadamard factorization of Hurwitz and Schur stable polynomials. In: R. Jeltsch and M. Mansour (editors), Stability Theory, Birkhäuser, 19-21, 1996.

[6] J. Garloff and J.D.G. Wagner. Hadamard product of stable polynomials are stable. J. Math. Anal. Appl., 202:797-808, 1996.

[7] J.H.B. Kemperman. A Hurwitz matrix is totally positive. SIAM J. Math. Anal., 13(2):331-341, 1982.

[8] C.A. Loredo-Villalobos and B. Aguirre-Hernández. Hadamard factorization of stable polynomials. Dyn. Contin. Discrete Impuls. Syst. A: Math. Anal., 22:325-332, 2015.

[9] C.A. Loredo-Villalobos and B. Aguirre-Hernández. Necessary conditions for Hadamard factorizations of Hurwitz polynomials. Automatica, 47:1409-1413, 2011. 